\title{
"Slow" Light in Media of "Zero" Dimension
}

\author{
Nikitas Papasimakis, Vassili A. Fedotov and Nikolay I. Zheludev \\ EPSRC NanoPhotonics Portfolio Centre, Optoelectronics Research Centre, University of Southampton, SO17 1BJ, UK \\ Sergey L. Prosvirnin \\ Institute of Radio Astronomy, National Academy of Sciences of Ukraine, Kharkov, 61002, Ukraine \\ Email: N.Papasimakis@soton.ac.uk
}

\begin{abstract}
Electromagnetic pulses propagating through a thin metal film, structured on the sub-wavelength scale, are significantly delayed and re-shaped.
\end{abstract}

For the first time, we provide experimental evidence that an electromagnetic pulse can be delayed by propagating through a planar meta-material, substantially thinner than the wavelength of radiation. The changes in the velocity and shape of light pulses propagating through bulk dispersive media have been studied extensively in the past [1,2]. In particular, it has been shown that the propagation velocity can be greater or smaller than in vacuum without significant change in the pulse shape and width. Recently, these effects have drawn considerable attention, since the ability to accurately control the velocity of light is essential for the development of optical communication technologies. To this end, a wide variety of methods have been suggested, such as, for example, electromagnetically induced transparency in atom vapors, coupled optical resonator waveguides and line defects in photonic crystals. However, the implementation of these approaches often requires special conditions (low temperature, laser pumping etc) and/or the size of the medium is comparable to the wavelength. We demonstrate that the pulse velocity and shape can be independently controlled by appropriate subwavelength patterning of a metal-dielectric interface, namely a planar metamaterial.

We observed electromagnetic pulse retardation and reshaping in a planar "fish-scale" structure [3], consisting of a periodic array of continuous wavy metallic strips with a $15 \times 15 \mathrm{~mm}$ unit cell residing on a $1.5 \mathrm{~mm}$ thick PCB dielectric substrate (see inset to Fig. 1a). This planar metamaterial exhibits frequency selective transmission accompanied by strong dispersion properties in the $\mathrm{GHz}$ frequency regime, as it can also be seen from the experimental spectrum for one polarization in Fig. 1a.

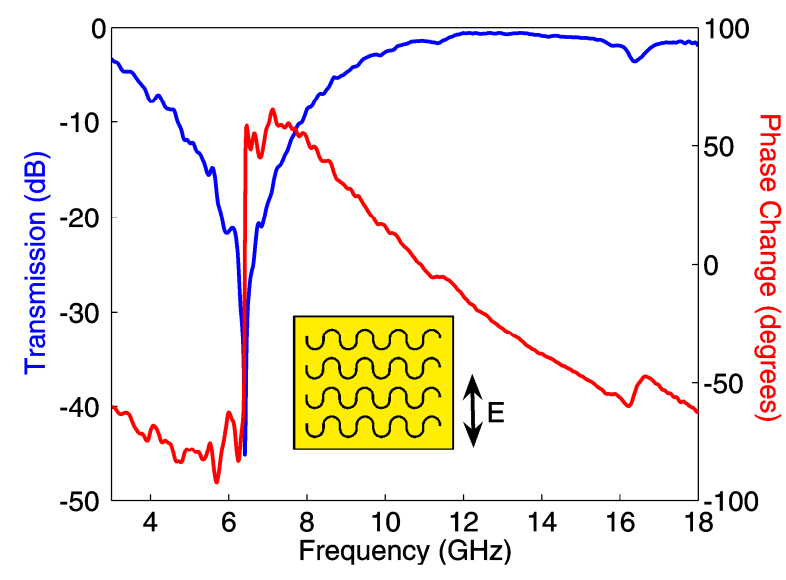

(a)

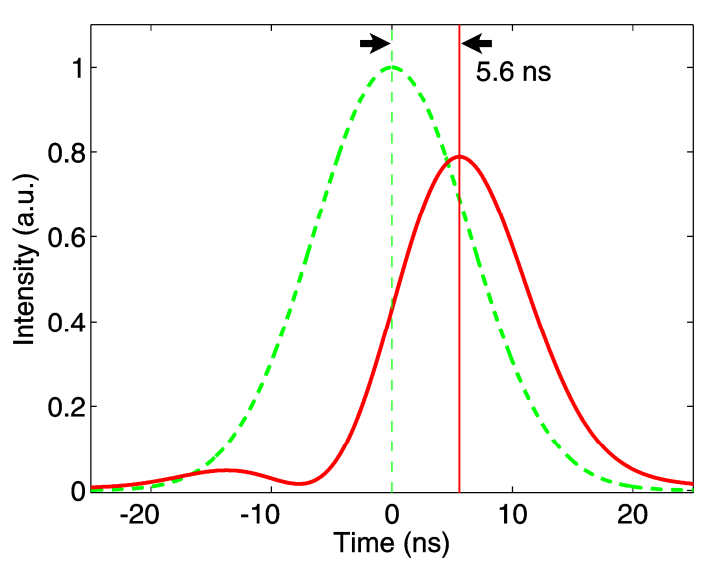

(b)

Fig. 1: (a) Amplitude (blue) and phase (red) transmission spectrum for the "fish scale" metamaterial shown in the inset. (b) Response (solid red, rescaled by $10^{4}$ ) of the fish scale structure to a $18 \mathrm{~ns}$ long pulse (dashed green).

By employing the inverse Fourier transform, the response of the metamaterial to microwave pulses can be reconstructed from the experimental spectrum of Fig. 1a. As examples of the structure's response to Gaussianshaped pulses, we consider $280 \mathrm{ps}$ short and $18 \mathrm{~ns}$ long pulses with center frequency in the resonant band ( 6.4 $\mathrm{GHz}$ ). A $18 \mathrm{~ns}$ long incoming pulse has a Fourier spectrum much narrower than the width of the resonance and therefore experiences a time delay of about $5.6 \mathrm{~ns}$ or roughly $1 / 3$ of the pulse width (see Fig. 1b). Such a delay is 
remarkable in view of the vanishing thickness of the metamaterial and corresponds to 10 pulse widths per wavelength. Moreover, the pulse retains to a great extent its initial Gaussian shape, with the exception of a very weak secondary advanced peak, while its width is slightly compressed. On the other hand, if a short pulse with approximately the same center frequency is considered, then two transmitted pulses emerge, traveling slower and faster than the incoming pulse, respectively. The two pulses have roughly the same amplitude and width and are well separated, while their delay and advance is about 100 ps and 190 ps, respectively (see Fig. 2). This phenomenon can be further exploited, if the propagation of a train of pulses is considered instead. In the inset of Fig. 2, we present the response of the metamaterial to a train of $280 \mathrm{ps}$ short Gaussian pulses with a 600 ps period, where a doubling of the modulation frequency is observed. This behavior, which is reminiscent of non-linear pulse splitting effects [4], can be of great practical interest in multiplexing and pulse compression systems, where control over the modulation frequency is required. Furthermore, the sub-wavelength thickness of the structure allows the succesive stacking of planar fish-scale slabs, in order to achieve virtually any desirable delay, while in the case of short pulses, this can result in a continuous response at the carrier frequency, always at the expense of pulse intensity.

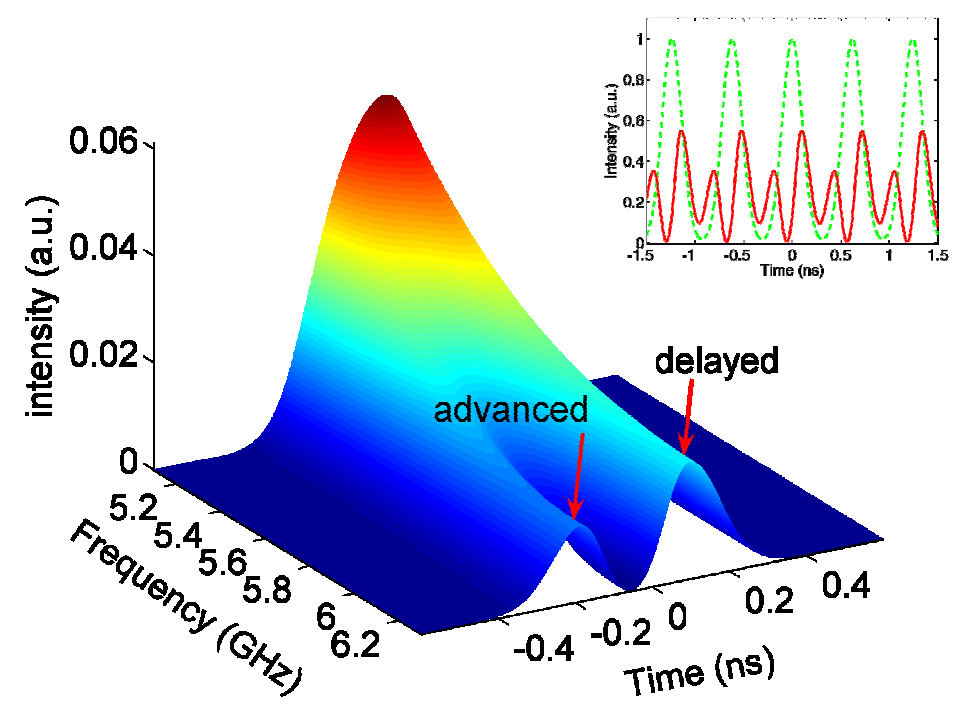

Fig. 2: Splitting of a $280 \mathrm{ps}$ short pulse with center frequency near the resonance. Inset: Doubling of the modulation frequency (solid red, rescaled by 25 ) of 280 ps short pulses (dashed green) after propagation through the fish scale structure.

In conclusion, we have shown that the propagation velocity and shape of microwave pulses can be controlled by introducing a planar metamaterial of "zero" dimension along the propagation direction. Unlike other approaches, the suggested configuration has vanishing thickness and can be easily manufactured by well developed planar lithography methods. Moreover, the use of geometrical resonances enables wide scalability and allows the structure to operate at prescribed frequencies. Such properties render planar metamaterials of this type, potentially useful as ultra-compact optical components with diverse functions, ranging from delay lines to pulse compression.

[1] L. Brillouin, Wave Propagation and Group Velocity (Academic Press Inc., New York, 1960).

[2] C. G. B. Garrett and D. E. McCumber, "Propagation of a Gaussian Light Pulse through an Anomalous Dispersion Medium," Phys. Rev. A 1, 305-313 (1970).

[3] V. A. Fedotov, P. L. Mladyonov, S. L. Prosvirnin, and N. I. Zheludev, "Planar electromagnetic metamaterial with a fish scale structure," Phys. Rev. E 72, 056613 (2005).

[4] J. E. Rothenberg, "Space-time focusing: breakdown of the slowly varying envelope approximation in the self focusing of femtosecond pulses”, Opt. Lett. 17, 1340-1342 (1992). 